\title{
Vida útil e qualidade de frutos de tomateiros tratados com paclobutrazol
}

\author{
Alexsander Seleguini ${ }^{1}$, Max José de Araújo Faria Júnior², Shizuo Seno, Jacira Isepon dos Santos, \\ Katiane Santigo da Silva 6 , Odair Lacerda Lemos ${ }^{7}$
}

\section{RESUMO}

A aplicação de reguladores de crescimento pode influenciar todas as fases do desenvolvimento de uma planta, incluindo o florescimento, o desenvolvimento e a maturação de frutos e sementes. Assim, avaliaram-se as principais características físicas e químicas e a vida útil dos frutos de tomate obtidos de plantas tratadas com paclobutrazol - PBZ (0, 50 e $\left.100 \mathrm{mg} \mathrm{L}^{-1}\right)$, via rega de mudas, aos 15 dias após a semeadura. Os frutos foram analisados imediatamente após a colheita e aos cinco, 10, 15 e 20 dias de armazenamento, em condições ambientes. A eficiência dos tratamentos e a qualidade dos frutos foram determinadas por meio da perda de matéria fresca, firmeza da polpa, teores de sólidos solúveis (SS), acidez titulável (AT), vitamina C e relação SS/AT. Adotou-se o delineamento experimental inteiramente casualizado, em esquema fatorial 3 x 5 (três concentrações de paclobutrazol x cinco períodos de armazenamento em temperatura ambiente). As aplicações de paclobutrazol em mudas de tomateiro, aos 15 dias após a semeadura, nas concentrações de 50 e $100 \mathrm{mg} \mathrm{L}^{-1}$, não alteraram a vida útil dos frutos do tomate longa vida AF 7631. O aumento das concentrações de PBZ resultou em menor perda de matéria fresca dos frutos e sua menor firmeza e acidez. Os teores de sólidos solúveis e de vitamina $\mathrm{C}$ e a relação SS/AT não se alteraram com o aumento das concentrações de PBZ. Os frutos, independentemente das concentrações de PBZ aplicadas às mudas, chegaram aos 20 dias de armazenamento $\left(25 \pm 2{ }^{\circ} \mathrm{C} \mathrm{e} 75 \pm 5 \%\right.$ UR) em condições para consumo, mesmo tendo perdido significativa quantidade de matéria fresca, acidez e firmeza.

Palavras-chave: Solanum lycopersicum L., regulador de crescimento, maturação.

\section{ABSTRACT}

\section{Shelf life and quality of tomato fruits from plants treated with paclobutrazol}

The application of growth regulators can influence all plant developmental phases, including flowering, development and maturation of fruits and seeds. In this study, we evaluated the physical and chemical characteristics and shelf life of tomato fruits from plants treated with Paclobutrazol - PBZ (0, 50 and $\left.100 \mathrm{mg} \mathrm{L}^{-1}\right)$ in drench application on seedlings, at 15 days after sowing. The fruits were analyzed immediately after harvest and after 5, 10, 15 and 20 days of storage in ambient conditions. The efficiency of treatments and fruit quality were determined by the parameters of weight loss,

Recebido para publicação em27/10/2009 e aprovado em 24/05/2011

'Engenheiro-Agrônomo, Doutor, Setor de Horticultura, Escola de Agronomia e Engenharia de Alimentos, Universidade Federal de Goiás, Campus Samambaia. Rodovia Goiânia / Nova Veneza, Km 0 - Caixa-Postal 131, 74001-970 Goiânia, GO, Brasil. aseleguini@gmail.com (Autor correspondente).

${ }^{2}$ Engenheiro-Agrônomo, Doutor, Departamento de Apoio, Produção e Saúde Animal, Faculdade de Odontologia de Araçatuba, Universidade Estadual Paulista. Rua Clóvis Pestana, 793; Dona Amélia, 16050-680, Araçatuba SP, Brasil. maxfaria@fmva.unesp.br

${ }^{3}$ Engenheiro-Agrônomo, Doutor, Departamento de Fitotecnia Tecnologia de Alimentos e Sócio Economia, Faculdade de Engenharia de Ilha Solteira, Universidade Estadual Paulista Júlio de Mesquita Filho. Av. Brasil, 56 - Centro, Caixa-Postal: 64, 15385-000 Ilha Solteira, SP, Brasil. shizuo@agr.feis.unesp.br

${ }^{4}$ Veterinária, Doutora, Departamento de Fitotecnia Tecnologia de Alimentos e Sócio Economia, Faculdade de Engenharia de Ilha Solteira, Universidade Estadual Paulista. Av. Brasil, 56 - Centro, Caixa-Postal: 64, 15385-000 Ilha Solteira, SP, Brasil. jacira@agr.feis.unesp.br

${ }^{5}$ Engenheira-Agrônoma, Doutora, Unidade Universitária de Aquidauana, Universidade Estadual de Mato Grosso do Sul. Rodovia Aquidauana/UEMS - Km 12, Zona Rural, 79200000, Aquidauana, MS, Brasil. kasantiago@ig.com.br

${ }^{6}$ Engenheiro-Agrônomo, Doutor, Departamento de Fitotecnia e Zootecnia, Universidade Estadual do Sudoeste da Bahia. Estrada do Bem Querer, Km 04 - Caixa-Posta 95, 45000000 Vitória da Conquista, BA, Brasil. odairlacerda@hotmail.com 
firmness, soluble solids (SS), titratable acidity (TA), vitamin C and SS/TA ratio. The experiment was arranged in a $3 \times 5$ factorial completely randomized design (concentrations of paclobutrazol x days of storage). The applications of paclobutrazol on tomato seedlings, 15 days after sowing, the concentrations of 50 and $100 \mathrm{mg} \mathrm{L}^{-1}$ had no effect on the fruit shelf-life of tomato cv. AF 7631. Increasing concentrations of PBZ resulted in lower weight loss, firmness and acidity of fruits. Soluble solids, vitamin $\mathrm{C}$ and SS/TA ratio did not change with increasing concentrations of PBZ. Fruits, regardless of the PBZ concentrations applied to seedlings, reached twenty days of storage $\left(25 \pm 2{ }^{\circ} \mathrm{C}\right.$ and $75 \pm 5 \%$ UR) fit for consumption, although with significant loss of fresh mass, acidity and firmness.

Key words: Solanum lycopersicum L, growth regulator, maturation.

\section{INTRODUÇÃO}

A aplicação de reguladores de crescimento influencia todas as fases do desenvolvimento de uma planta, incluindo o florescimento, o desenvolvimento e a maturação de frutos e sementes (Han \& Kim, 1999). Algumas pesquisas têm demonstrado a eficiência da aplicação foliar ou via solo de Paclobutrazol (PBZ) na redução do crescimento e desenvolvimento vegetativo de tomateiros (Asao et al. 1996; Berova \& Zlatev, 2000; Giovinazzo \& Souza-Machado, 2001; Nascimento et al. 2003; Silva e Faria Jr., 2011), com reflexos também no florescimento (Berova \& Zlatev, 2000; Souza-Machado et al., 1999; Seleguini, 2007) e na produtividade (Berova \& Zlatev, 2000; Giovinazzo \& Souza-Machado, 2001; Seleguini, 2007), todavia os efeitos na qualidade dos frutos ainda não estão claros.

Segundo Fletcher et al. (2000), triazóis como o PBZ, inibem a conversão de ent-caureno para ácido entcaurenoico na biossíntese de ácido giberélico (GA), que resulta em redução nos níveis de todas as formas de GAs, causando reduções na taxa de elongação e divisão celular, sem ocasionar citotoxicidade. Existem relatos que indicam que o PBZ pode alterar os níveis de outros hormônios, como o ácido abscísico, o etileno, as citocininas (Fletcher et al., 2000) e auxinas (Davis \& Curry, 1991), além de outras características fisiológicas, como a síntese de clorofila (Berova \& Zlatev, 2000; Still \& Pill, 2004) e a partição de fotoassimilados (Tekalign \& Hammes, 2005). Diante disso, a aplicação do PBZ poderia, indiretamente, influenciar algumas das qualidades físicas e químicas e a vida útil de frutos produzidos nas plantas tratadas. Além disso, segundo Hamid \& Williams (1997) e Quinlan \& Richardson (1986), o PBZ absorvido pelas raízes é translocado, principalmente, via fluxo transpiracional, pelo xilema. Contudo, estudos com Pistachia chinensis e Ricinus communis (Witchard, 1997 a, b) sugeriram que poderia existir um mecanismo secundário de transporte floemático, que poderia levar o produto aos frutos.
Os resultados de pesquisas existentes quanto à influência da aplicação do PBZ na qualidade de frutos são poucos e restritos à espécies de porte arbóreo. Yeshitela et al. (2004) verificaram aumentos significativos no teor de sólidos solúveis totais, açúcares redutores e açúcar total, aliados à redução de acidez de frutos de mangueira Tommy Atkins tratada com PBZ, em comparação a frutos de plantas não tratadas. Em maçãs produzidas em árvores tratadas com PBZ, Khurshid et al. (1997) verificaram aumentos não significativos no teor de sólidos solúveis, estimados em $7 \%$ no primeiro ano e $11 \%$ no segundo. Também a firmeza da polpa foi aumentada em frutos oriundos de plantas tratadas com PBZ. Christov et al. (1995) também verificaram melhorias significativas na qualidade de uvas das plantas tratadas com PBZ, com aumentos de até $10 \%$ no teor de sólidos solúveis totais e reduções de até $20 \%$ na acidez. Jacyna \& Dodds (1995), por exemplo, não verificaram alterações na qualidade de frutos no que tange aos níveis de sólidos solúveis, acidez e firmeza da polpa de frutos de damasqueiro tratados com PBZ. O mesmo foi obtido por Curry \& Williams (1986) com frutos de maçã e pêra e por Mendonça et al. (2002) com manga.

Outro ponto importante que pode estar relacionado com a manutenção da qualidade dos frutos é a ação antifúngica atribuída ao PBZ, uma vez que o mesmo é estruturalmente similar aos fungicidas do grupo triazol (Deas \& Clifford, 1984).

Em plantas anuais, as pesquisas não têm dado importância aos efeitos do emprego do PBZ sobre os atributos físico-químicos de qualidade dos frutos produzidos. Em tomateiros, esse aspecto ganha importância, uma vez que o tomate é um fruto climatérico e apresenta modificações expressivas durante o amadurecimento, que culmina na alteração da cor, na firmeza da polpa, no flavor e no aroma. Além disso, o etileno, fito-hormônio do amadurecimento, está diretamente envolvido no desenvolvimento desses eventos.

Rev. Ceres, Viçosa, v. 58, n.4, p. 470-475, jul/ago, 2011 
Este estudo teve como objetivo verificar o efeito do tratamento de plantas com concentrações crescentes de PBZ aplicadas em mudas aos 15 dias após a semeadura, no armazenamento e nas características físicas e químicas de frutos de tomateiro longa vida, híbrido AF 7631.

\section{MATERIAL E MÉTODOS}

O experimento foi conduzido no período de setembro de 2006 a fevereiro de 2007 na Faculdade de Engenharia, UNESP, Campus de Ilha Solteira, SP, situada a $20^{\circ} 22^{\prime}$ S, $51^{\circ} 22^{\prime}$ e altitude de $330 \mathrm{~m}$. As análises foram realizadas no Laboratório de Tecnologia de Alimentos durante o mês de fevereiro de 2007.

Fez-se o cultivo do tomateiro em ambiente protegido, utilizando o híbrido tipo longa vida AF 7136, da empresa Sakata Seed Sudamerica. As sementes foram semeadas em 21/09/2006 em bandejas contendo substrato comercial PLANTMAX $^{\circledR}$. Os tratamentos das mudas com PBZ nas concentrações de 0,50 e $100 \mathrm{mg} \mathrm{L}^{-1}$ foram realizados aos 15 dias após semeadura (DAS), por meio da aspersão de 5 mL de solução de PBZ por muda, sendo o volume suficiente para bom molhamento das folhas e do substrato. $\mathrm{O}$ transplantio foi realizado aos 34 DAS (25/10/2006).

A colheita foi realizada de forma manual, aos 100 dias após o transplantio, retirando-se o pedúnculo e os cálices para que não houvesse lesões por atrito. $\mathrm{O}$ critério utilizado para determinar o ponto de colheita dos frutos foi a coloração da epiderme. Após a colheita, os frutos dos três tratamentos foram transportados ao Laboratório de Tecnologia de Alimentos, onde passaram por limpeza com o auxílio de um pano seco, para retirada de umidade e outros resíduos superficiais. Os frutos passaram também por seleção manual, descartando-se aqueles portadores de imperfeições facilmente detectáveis e desuniformidade de maturação, dando preferência a frutos pintados (com ápice amarelecendo) e coloridos (tomate com a cor entre pintado e com mais de $90 \%$ da cor final), de acordo com as normas do Programa Brasileiro para Modernização da Horticultura (Companhia de Entrepostos e Armazéns Gerais de São Paulo - CEAGESP, 2003). Em seguida, os frutos foram colocados em bandejas de papelão, onde foram mantidos em condições ambiente (temperatura de $26 \pm 3{ }^{\circ} \mathrm{C}$ e umidade relativa do ar de $75 \pm 5 \%$ ) até o final do experimento.

Os frutos foram analisados imediatamente após a colheita e após cinco, 10, 15 e 20 dias de vida útil, em condições ambientais. A eficiência dos tratamentos e a qualidade dos frutos foram determinadas pela matéria fresca e firmeza da polpa; e parâmetros químicos: acidez titulável (AT), teores de sólidos solúveis (SS), vitamina C e relação entre SS/AT.
O delineamento experimental utilizado foi o inteiramente casualizado, em esquema fatorial 3 x 5 (três concentrações de PBZ x cinco períodos de armazenamento), com três repetições, no qual cada repetição consistiu de três frutos. Os dados obtidos foram submetidos à análise de variância (teste F), e para as médias dos fatores foram ajustadas equações de regressão polinomial.

A perda percentual de matéria fresca foi determinada por meio da diferença entre a matéria fresca inicial dos frutos nas unidades experimentais e a matéria fresca no dia da amostragem, em relação à matéria fresca inicial. A firmeza da polpa foi medida em dois lados opostos da superfície longitudinal de cada fruto, pela utilização de um penetrômetro modelo FT 327 com ponteira de $8 \mathrm{~mm}$, e os valores foram expressos em libras.

A acidez titulável (AT), expressa em gramas de ácido cítrico por $100 \mathrm{~g}$ de polpa, e o teor de vitamina $\mathrm{C}$, expresso em $\mathrm{mg}$ de ácido ascórbico por $100 \mathrm{~g}$ de polpa, foram determinados por titulometria de acordo com as normas do Instituto Adolfo Lutz (1985). Os teores de sólidos solúveis (SS), expressos em ${ }^{\circ}$ Brix, foram determinados por refratometria.

\section{RESULTADOS E DISCUSSÃO}

Não houve efeito da interação entre os fatores concentrações de PBZ e período de armazenamento sobre nenhuma das características químicas e físicas avaliadas.

Independentemente do período de armazenamento, a perda de massa da matéria fresca, a firmeza de polpa e a acidez titulável (AT) de frutos foram alteradas significativamente com o aumento das concentrações do PBZ aplicadas nas mudas (Figura $1 \mathrm{a}, \mathrm{b}, \mathrm{c}$ ). Por sua vez, os teores de sólidos solúveis, vitamina $\mathrm{C}$ e a relação SS/AT não foram influenciados pelas concentrações de PBZ, cujos valores médios situaram em 4,1 ${ }^{\circ} \mathrm{Brix}, 27,52$ e 19,82 mg de ácido ascórbico $100 \mathrm{~g}$ de polpa $^{-1}$, respectivamente. Frutos produzidos de plantas tratadas com 50 e $100 \mathrm{mg} \mathrm{L}^{-1}$ de PBZ apresentaram menor perda de massa da matéria fresca e menor acidez comparados aos obtidos de plantas sem tratamento com PBZ (Figura 1 a, c). Já para firmeza de polpa constatou-se que frutos oriundos de plantas-controle, sem aplicação de PBZ, apresentaram maior firmeza que os frutos de plantas tratadas com 50 e $100 \mathrm{mg} \mathrm{L}^{-1}$ (Figura 1 b).

Asao et al. (1996) também não observaram alterações no teor de sólidos solúveis em frutos de tomateiro quando as mudas com duas folhas verdadeiras foram tratadas via pulverização foliar com PBZ (12,5 e $\left.100 \mathrm{mg} \mathrm{L}^{-1}\right)$. Já Ramirez et al. (2005) verificaram que a aplicação, em tomateiro com 12 folhas verdadeiras, de outro inibidor da biossíntese de ácido giberélico, a prohexadiona cálcio, proporcionou aumentos no teor de sólidos solúveis totais e na firmeza do fruto. 
Reduções no nível de acidez de frutos também foram verificadas em manga (Yeshitela et al., 2004), e em uva (Christov et al., 1995); entretanto, em ambos os experimentos foram observados aumentos no teor dos sólidos solúveis. Khurshid et al. (1997) notaram incrementos não significativos no teor de sólidos solúveis e aumento na firmeza de frutos de maçã oriundos de plantas tratadas com PBZ. Jacyna \& Dodds (1995) não verificaram alterações na qualidade de frutos no que tange aos níveis de sólidos solúveis, acidez e firmeza da polpa de frutos de damasqueiro tratados com PBZ. O mesmo foi observado por Curry \& Williams (1986), em frutos de maçã e pera, e por Mendonça et al. (2002), em manga.

Os possíveis efeitos atribuídos ao PBZ sobre a qualidade de frutos verificada no presente trabalho podem ter
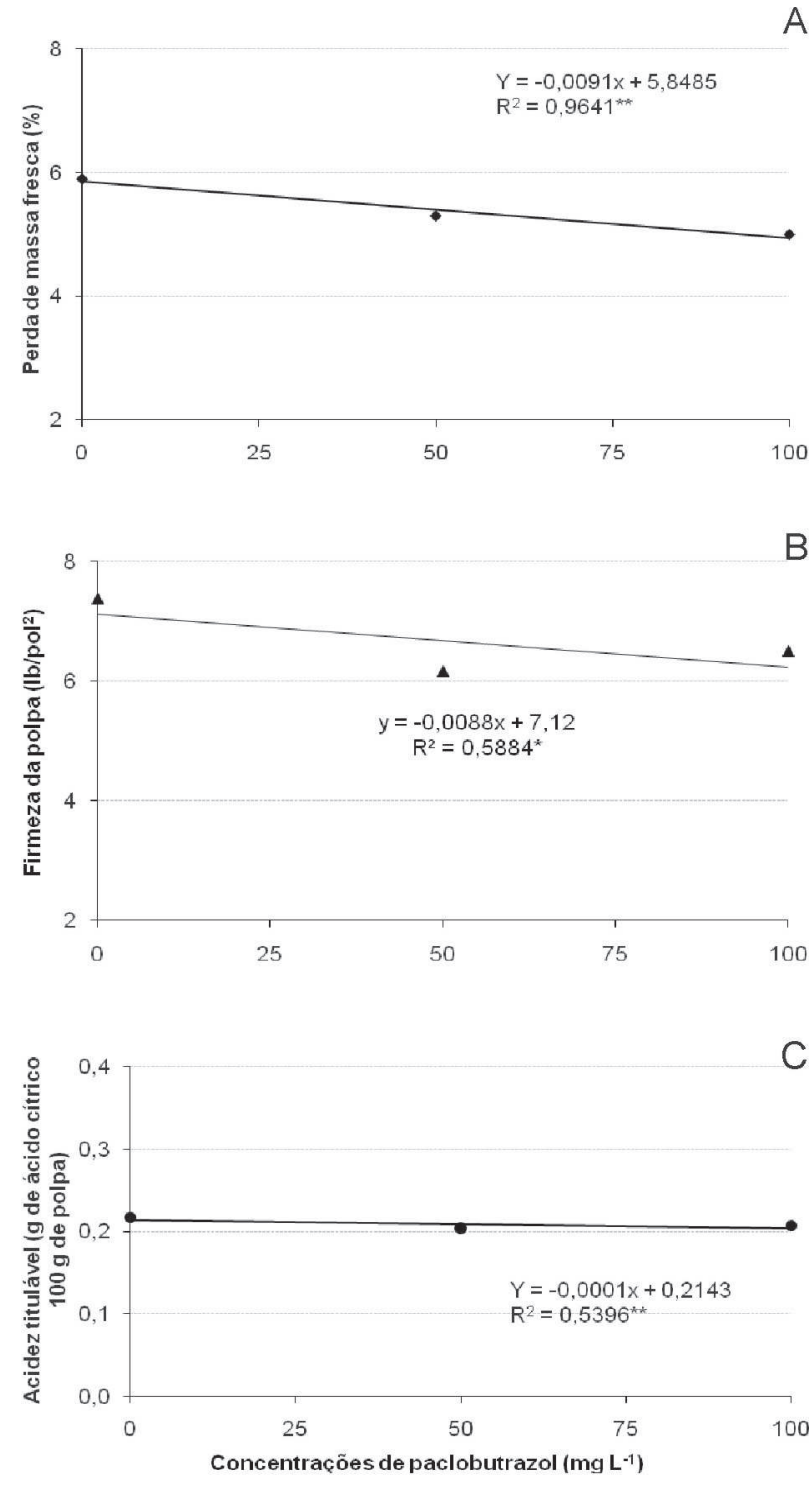

Figura 1. Perda de massa da matéria fresca (a), firmeza de polpa (b) e acidez titulável (c) de frutos de tomate longa vida AF 7631 em função de concentrações de paclobutrazol, aplicado aos 15 dias após a semeadura, via rega de mudas. sido ocasionados, indiretamente, por modificações na fisiologia da planta. Yeshitela et al. (2004) sugeriram que as alterações ocorridas na qualidade de frutos de manga Tommy Atkins proporcionadas pelo uso do PBZ podem estar relacionadas com a partição de assimilados na planta, uma vez que a demanda assimilativa é unidirecional ao fruto em desenvolvimento. Por causa da grande supressão do crescimento vegetativo, as árvores tratadas tiveram os atributos de qualidade melhorados.

Os efeitos diretos do PBZ nos frutos provavelmente seriam maiores se a aplicação fosse dirigida aos frutos ou se as quantidades expressivas fossem translocadas aos frutos. Entretanto, a translocação dos triazóis é principalmente via xilema, e a análise de resíduo em frutos de plantas tratadas com PBZ evidencia isso. Prive et al. (1989) verificaram que os resíduos de $\mathrm{PBZ}$ em frutos de maçãs tratadas com PBZ sempre ficaram abaixo dos limites detectáveis $\left(0,01 \mathrm{mg}\right.$ g matéria fresca $\left.{ }^{-1}\right)$. Berova \& Zlatev (2000) observaram que somente $0,007 \mu \mathrm{g}$ de PBZ g de matéria fresca ${ }^{-1}$ foi encontrada em frutos de tomate desenvolvidos em plantas tratadas com $1 \mathrm{mg} \mathrm{L}^{-1}$ de PBZ aplicado via rega do solo, no dia do transplantio.

Quanto ao período de armazenamento pós-colheita, verificou-se aumento na perda de massa (Figura 2 a); ou seja, houve redução significativa na massa da matéria fresca dos frutos. A firmeza de frutos reduziu linearmente ao longo do armazenamento (Figura 2 b). Após 20 dias de armazenamento, a perda estimada de massa da matéria fresca de frutos foi de $11 \%$ e a de firmeza, de $21 \%$. Segundo Bhowmik \& Pan (1992), a perda de massa do tomate durante o armazenamento ocorre principalmente devido a dois fatores: a transpiração e a respiração. A transpiração, que é a maior responsável pela perda de massa, é o mecanismo pelo qual a água é perdida devido à diferença de pressão de vapor d'água entre a atmosfera circundante e a superfície do fruto. De acordo com Chitarra \& Chitarra (1990), a perda de firmeza do fruto é devida a alterações nas características dos polissacarídeos da parede celular, cujos principais componentes são substâncias pécticas.

O teor de sólidos solúveis aumentou linearmente com o tempo de armazenamento. Partindo de um teor médio de 3,9 ${ }^{\circ}$ Brix no dia da colheita, alcançou $4,1^{\circ}$ Brix após 20 dias de armazenamento em condições ambiente (Figura 2 c). Esse comportamento pode ser explicado tanto pela hidrólise do amido a açúcares redutores (frutose e glicose), que ocorre durante o amadurecimento, como pela perda de água que contribui para a concentração dos sólidos solúveis.

A acidez titulável reduziu linearmente com o aumento do período de armazenamento. O teor observado no dia da colheita, de 0,234\% de ácido cítrico na polpa, decaiu a 0,198\% após 20 dias de armazenamento, o que representou redução de mais de $15 \%$ na quantidade de ácido cítrico (Figura 2 d). 

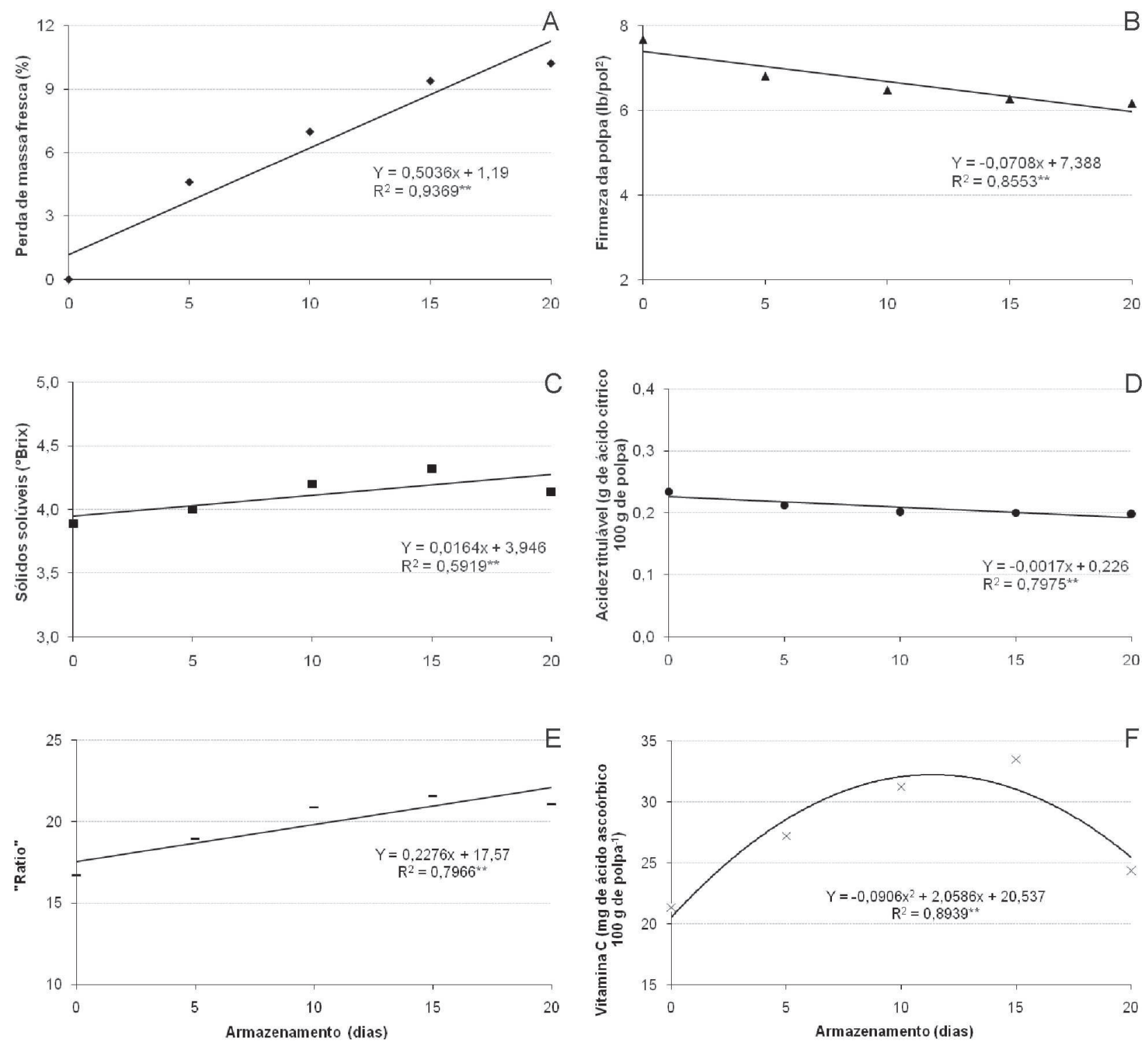

Figura 2. Perda de massa da matéria fresca (a), firmeza de polpa (b), teores de sólidos solúveis (c), acidez titulável (d), relação SS/AT (e) e teor de vitamina C (f) de frutos de tomate longa vida AF 7631 em função do período de armazenamento em temperatura ambiente.

Essa redução ocorreu devido à característica climatérica do tomate, que faz com que os ácidos orgânicos sejam os primeiros substratos utilizados na respiração (Chitarra \& Chitarra, 1990).

O aumento dos sólidos solúveis e a redução da acidez com o aumento do armazenamento possibilitaram aumento significativo na relação SS/AT, o que tem sido correlacionado com o índice de sabor dos frutos (Saimbhi et al., 1995). Segundo Kader et al. (1978), o tomate para consumo in natura é considerado de excelente 'sabor' quando apresenta relação SS/AT superior a 10. Para todas as avaliações, as relações foram superiores a 10 (Figura 2 e); estando, portanto, os tomates adequados ao consumo in natura.

Houve resposta de forma quadrática do teor de vitamina C nos frutos, sendo a concentração máxima de 32 mg estima- da aos 11 dias de armazenamento (Figura 2 f). Segundo Hobson \& Davies (1971), os dados sobre o comportamento do ácido ascórbico durante a maturação de tomate são inconsistentes. Alguns autores afirmam que há pouca mudança no teor desse ácido, enquanto outros, como Dalal et al. (1965), evidenciaram aumento do teor de ácido ascórbico com a maturação do fruto.

\section{CONCLUSÕES}

$\mathrm{O}$ aumento das concentrações de PBZ resulta em menor perda de matéria fresca dos frutos e menor firmeza e acidez.

$\mathrm{O}$ aumento das concentrações de PBZ não altera o teor de sólidos solúveis, vitamina $\mathrm{C}$ e a relação SS/AT em frutos de tomate 
Os tomates, independentemente das concentrações de PBZ, chegaram aos 20 dias de armazenamento $(26 \pm 2$ ${ }^{\circ} \mathrm{C}$ e $75 \pm 5 \%$ UR) em condições de consumo, mesmo tendo perdido considerável matéria fresca, acidez e firmeza.

\section{REFERÊNCIAS}

Asao T, Ito N, Hosoki T, Ohta K \& Endo K (1996) Effects of plant growth retardants and root pruning on growth and yield of tomato cultured hydroponically at high temperature during summer. Journal of the Japanese Society for Horticultural Science, 65:89-94.

Berova M \& Zlatev Z (2000) Physiological response and yield of paclobutrazol treated tomato plants (Lycopersicon esculentum Mill.). Plant Growth Regulation, 30:117-123.

Bhowmik SR \& Pan AJC (1992) Shelf life of mature green tomatoes stored in controlled atmosphere and high humidity. Journal of Food Science, 57:948-953.

Chitarra MIF \& Chitarra AB (1990) Pós-colheita de frutas e hortaliças: fisiologia e manuseio. Lavras, ESAL/FAEPE. 293p.

Christov C, Tsvetkov I \& Kovachev V. (1995) Use of paclobutrazol to control vegetative growth and improve fruiting efficiency of grapevines (Vitis vinifera 1.). Bulgarian Journal of Plant Physiology, 21:64-71.

Companhia de Entrepostos e Armazéns Gerais de São Paulo Ceagesp (2003) Programa brasileiro para modernização da horticultura. Normas de classificação do tomate: Centro de Qualidade em Horticultura. São Paulo, CQH/CEAGESP. (CQH. Documentos, 26).

Curry EA \& Williams MW (1986) Effect of paclobutrazol on fruit quality: apple, pear and cherry. Acta Horticulturae, 179:743753

Dalal KB, Salunkhe DK, Boe AA \& Olson LE (1965) Certain physiological and biochemical changes in the developing tomato fruit (Lycopersicon esculentum Mill). Journal of Food Science, 30:504-508.

Davis T \& Curry E (1991) Chemical regulation of vegetative growth. Critical Reviews in Plant Sciences, 10:151-158.

Deas AHB \& Clifford DR (1984) Reductive and oxidative metabolism of triazolymethanes by two basidiomycete fungi. Pesticide Biochemistry and Physiology, 22:276-284.

Fletcher RA, Gilley A, Sankhla N \& Davis T (2000) Triazoles as plant growth regulators and stress protectants. Horticultural reviews, 24:55-138.

Giovinazzo R \& Souza-Machado V (2001) Paclobutrazol responses with processing tomato in France. Acta horticulturae, 542:355358

Hamid MM \& Williams RR (1997) Effect of different types and concentrations of plant growth retardants on Sturt's desert pea (Swainsona formosa). Scientia Horticulturae, 71:79-85.

Han IS \& Kim JG (1999) Effects of growth regulators on growth, flowering, and germination of harvested seeds in Clinopodium chinense var. parviflorum. Journal of the Korean Society for Horticultural Science, 40:765-768.

Hobson GE \& Davies JN (1971) The tomato. In: Hulme, AC (Ed.) The Biochemistry of fruits and their products. London. Academic Press, p.437-475.

Instituto Adolfo Lutz (1985) Normas analíticas do Instituto Adolfo Lutz: métodos químicos e físicos para análise de alimentos. $3^{\mathrm{a}}$ Ed. São Paulo. IMESP, 533p.
Jacyna T \& Dodds KG (1995) Some effects of soil-applied paclobutrazol on performance of 'Sundrop' apricot (Prunus armeniaca $\mathrm{L}$ ) trees and on residue in the soil. New Zealand Journal of Crop and Horticultural Science, 23:323-329.

Kader AA, Morris LL, Stevens MA \& Albright-Holton M (1978) Composition and flavor quality of fresh market tomatoes as influenced by some post harvest handling procedures. Journal of the American Society for Horticultural Science, 113:6-13.

Khurshid T, Mcneil DL, Trought MCT \& Hill GD (1997) The response of young 'Braeburn' and 'Oregon Spur Delicious' apple trees growing under an ultrahigh density planting system to soil-applied paclobutrazol II Effect on fruit quality at harvest and during storage. Scientia Horticulturae, 71:189-196.

Mendonça V, Araujo Neto SE, Ramos JD, Pio R \& Souza P (2002) A Produção e qualidade da manga 'Tommy Atkins' após aplicação de paclobutrazol. Revista de Ciências Agrárias, 2:39-149.

Nascimento, WM, Salvalagio, R; Silva, JBC (2003) Condicionamento químico do crescimento de mudas de tomate. Horticultura brasileira, 21 - suplemento cd.

Prive JP, Elfving DC \& Proctor JTA (1989) Paclobutrazol, gibberellin, and cytokinin effects on growth, development, and histology of apple pedicels and fruit. Journal of the American Society for Horticultural Science, 114:273-278.

Quinlan JD \& Richardson PJ (1986) Uptake and translocation of paclobutrazol and implications for orchard use. Acta Horticulturae, 179:443-451.

Ramirez H, Manjarrez RMP, Mendoza AB, López AS, Torres VR \& Davila JH (2005) Efectos de prohexadiona - Ca em tomate y su relación com la variación de la concetración de giberelinas y citocininas. Revista Chapingo Serie Horticultura, 11:283-290.

Saimbhi MS, Cheema DS, Singh S \& Nandpuri KS (1995) Physicochemical characteristics of some tomato hybrids. Tropical Sciences, 35:9-12.

Seleguini A (2007) Uso de paclobutrazol na produção de mudas, no crescimento, produção e qualidade de frutos de tomateiro em ambiente protegido. Universidade Estadual Paulista "Campus de Ilha Solteira", Ilha Solteira, 100p.

Silva KS, Faria Junior MJA (2011) Uso de paclobutrazol como estratégia para redução do porte e da brotação lateral de plantas de tomateiro. Ciência e Agrotecnologia, 35:539-546.

Souza-Machado V, Pitblado R, Ali A, May P (1999) Paclobutrazol in tomato (Lycopersicon esculentum) for improved tolerance to early transplanting and earlier harvest maturity. Acta Horticulturae, 487:139-143.

Still JR \& Pill WG (2004) Growth and stress tolerance of tomato seedlings (Lycopersicon esculentum Mill) in response to seed treatment with paclobutrazol Journal of Horticultural Science \& Biotechnology, 79:197-203.

Tekalign T \& Hammes PS (2005) Growth and biomass production in potato grown in the hot tropics as influenced by paclobutrazol. Plant Growth Regulation, 35:37-46.

Witchard MA (1997a) Simplified technique for detection of paclobutrazol in plant Ssp extracts, using HPLC. Journal of Plant Growth Regulation, 16:213-214.

Witchard MA (1997b) Paclobutrazol Is Phloem Mobile in Castor Oil Plant (Ricinus communis L). Journal of Plant Growth Regulation, 16:215-217.

Yeshitela T, Robbertse PJ \& Stassen PJC (2004) Paclobutrazol suppressed vegetative growth and improved yield as well as fruit quality of 'Tommy Atkins' mango (Mangifera indica) in Ethiopia. New Zealand Journal of Crop and Horticultural Science, $32: 281-293$.

Rev. Ceres, Viçosa, v. 58, n.4, p. 470-475, jul/ago, 2011 